U. Hegerl, M. Zaudig und H.J. Möller (Hrsg.)

Depression und Demenz im Alter. Abgrenzung,

Wechselwirkungen, Diagnose, Therapie

Wien, Springer, 2001, 162 S., € 61,-

ISBN 3-211-83569-5

Altersdepression und Demenz sind die häufigsten psychiatrischen Erkrankungen des höheren Lebensalters. Dies erscheint besonders vor dem Hintergrund der momentanen Bevölkerungsentwicklung, aber auch aus gesundheitspolitischer Perspektive relevant. Neue antidementive Behandlungsstrategien sind zum Teil sehr kostspielig und werden dementsprechend nur zurückhaltend verordnet.

Mit ihrem Buch möchten die Herausgeber den in diesem Arbeitsfeld tätigen Berufsgruppen diagnostische und therapeutische Entscheidungen durch fundiertes Wissen erleichtern. Die Vermittlung von ausführlichen Informationen zum Thema Demenz und Depression bezüglich ihres gemeinsamen Auftretens, ihrer Abgrenzung und ihrer Therapie ist den Autoren sehr gut gelungen. Dabei beleuchten sie zusätzlich die häufig sehr schwierig zu treffenden Differentialdiagnosen «Leichte kognitive Beeinträchtigung» und «Normales Altern». Das enge Zusammenspiel von Depression und Demenz und die damit verbundenen diagnostischen Probleme werden vor allem durch das Konzept herausgearbeitet, das die beiden Erkrankungen in jeweils gemeinsamen Kapiteln zu den Themen Epidemiologie, Diagnose und Differentialdiagnose gegenüberstellt. Positiv hervorzuheben ist auch, dass neben den üblichen medikamentösen Maßnahmen im Bereich Therapie auch kognitiv-verhaltenstherapeutischen Aspekten Platz eingeräumt wird.

Das Buch besteht aus den drei großen Themenbereichen Epidemiologie, Diagnostik und Therapie, die in insgesamt sieben Unterkapitel gegliedert sind. Die einzelnen Kapitelbeiträge wurden von unterschiedlichen Autoren aus verschiedenen Berufsgruppen verfasst. Dabei fällt auf, dass dem Thema Therapie im Vergleich zur Diagnostik mehr Platz eingeräumt wurde. Dies überrascht vor dem Hintergrund differentialdiagnostischer Probleme in Forschung und Praxis.

Der Themenbereich Epidemiologie von Depression und Demenz im Alter gibt einen umfassenden Einblick in die Erkrankungshäufigkeiten und berücksichtigt dabei auch methodische Schwierigkeiten epidemiologischer Studien. Diese Schwierigkeiten wurden hier auch bei der Auswahl repräsentativer Studien berücksichtigt. Der Leser bekommt an dieser Stelle bereits einen ersten Eindruck in die Problematik Depression in Abgrenzung zur Demenz - und ihre Komorbiditäten.
Der zweite Themenbereich Diagnostik beginnt mit einem allgemeinen Kapitel zur Diagnose und Differentialdiagnose von Depression und Demenz im Alter. Dabei werden klinische Gesichtspunkte, diagnostische Kriterien und deren psychometrische Erfassung übersichtlich dargestellt. Neben den beiden Hauptstörungen Depression und Demenz und deren Unterformen wird der «Leichten kognitiven Beeinträchtigung» (LKB) viel Platz eingeräumt. Deren differentialdiagnostische Abgrenzung zu Depression und Demenz stellt in der Praxis meist das größte diagnostische Problem dar. Patienten mit LKB tragen ein erhöhtes Risiko, im weiteren Verlauf an einer Demenz zu erkranken. Der Bedeutung ihrer Identifizierung wird an dieser Stelle Rechnung getragen.

Das folgende Kapitel beschäftigt sich mit den neuropsychologischen Auffälligkeiten im Rahmen des normalen Alterns, bei Depression und Demenz. An dieser Stelle wäre eine praxisbezogene Erläuterung nützlicher neuropsychologischer Untersuchungsverfahren der beschriebenen kognitiven Funktionen wünschenswert gewesen. Dies gelingt den Autoren des folgenden Kapitels zu biologischen Untersuchungen in der Differentialdiagnostik kognitiver und depressiver Störungen im Alter. Dabei werden aktuelle Ergebnisse routinemäßiger und fakultativer Untersuchungsverfahren zur Differentialdiagnostik diskutiert. Besonders gut werden hier neuere Befunde der in der Praxis immer häufiger eingesetzten elektrophysiologischen und bildgebenden Verfahren dargestellt. Für den interessierten Leser finden sich hier eine Reihe von Verweisen auf weiterführende Literatur zum Thema.

Der nächste große Themenbereich beschäftigt sich mit der Therapie von Depression und Demenz im Alter. Dabei werden im Gegensatz zu den vorhergehenden Kapiteln medikamentöse Therapien von Depression und Demenz sinnvollerweise in getrennten Kapiteln behandelt. Im Kapitel Antidepressiva wird zunächst eindringlich auf den Missstand nicht diagnostizierter und dementsprechend nicht therapierter depressiver Erkrankungen bei alten Menschen hingewiesen. Dass auch ältere Menschen von antidepressiver Behandlung profitieren, wird hier mit der ausführlichen Darstellung pharmakologischer Studien belegt. Einen besonderen und wichtigen Schwerpunkt dieses Kapitels stellt die Besonderheit medikamentöser Maßnahmen bei älteren depressiven Patienten dar. Aufgrund ihrer Multimorbidität und des veränderten Stoffwechsels müssen hier besondere Therapiestrategien verfolgt werden. Auch häufige Komedikationen bei komorbider Demenz oder Parkinson-Syndrom, nach Hirninsult und bei kardialen Störungen werden an dieser Stelle berücksichtigt. Das Kapitel endet mit prakti- 
schen Ratschlägen zur antidepressiven Behandlung älterer Menschen.

Das Kapitel Nootropika/Antidementiva konzentriert sich vor allem auf cholinerge Behandlungsstrategien, wobei jedoch auch die Wirksamkeit älterer Präparate wie z.B. Piracetam und Ginkgo-biloba-Extrakt betont wird. Mit dem Ziel einen Eindruck zu vermitteln, was unter den gegenwärtigen Bedingungen therapeutisch erreichbar ist, beginnt auch dieses Kapitel mit einer sehr ausführlichen Darstellung pharmakologischer Prüfstudien zu den einzelnen Substanzen. Das Kapitel verdeutlicht die Problematik antidementiver Medikamente, die aufgrund häufig schwer objektivierbarer Behandlungserfolge, fehlender Wirksamkeitskriterien und ihres begrenzten Nutzens von Praktikern nur zurückhaltend verordnet werden. Insgesamt veranschaulicht dieser Beitrag einerseits den Fortschritt antidementiver Behandlungskonzepte, zeigt aber andererseits auch den dringenden Bedarf für weitere Forschung in diesem Bereich auf. Das Kapitel endet mit einer Übersicht der gebräuchlichen Antidementiva und Nootropika.

In den beiden letzten Kapiteln des Bereichs Therapie werden Depression und kognitive Defizite wieder in jeweils gemeinsamen Beiträgen zusammengefasst. Kognitiv-verhaltenstherapeutische Interventionen werden bei älteren Menschen trotz erwiesener Wirksamkeit häufig vernachlässigt. Die praxisnahe Beschäftigung der Autoren mit diesem Thema mit Hilfe selbst erarbeiteter Programme ist an dieser Stelle deshalb besonders positiv hervorzuheben. Mit konkreten Handlungsanweisungen werden hier ein alltagsorientiertes Gedächtnis-Training, ein verhaltenstherapeutisches Kompetenztraining für Patienten mit beginnender Alzheimer-Demenz sowie ein psychoedukativer kognitiv-behavioraler Ansatz bei älteren depressiven Patienten vorgestellt. Das Kompetenztraining für Patienten mit beginnender Alzheimer-Demenz erscheint hierbei sehr umfangreich und ist in seiner Ganzheit wohl nicht bei allen Patienten durchzuführen. Es enthält aber wesentliche Bausteine, die auch isoliert betrachtet die Selbständigkeit der Patienten fördern dürften. Dies sind z.B. Psychoedukation für Patienten und Angehörige, Steigerung des Aktivitätsniveaus und die Berücksichtigung der häufig auftretenden depressiven Begleitsymptomatik. Insgesamt besticht das Kapitel durch die Verwendung ressourcenorientierter Ansätze, die gerade bei älteren Menschen im Hinblick auf Lebensqualität und Selbständigkeit bedeutsam sind.

Das letzte Kapitel beschäftigt sich mit sozialpsychiatrischen Aspekten der beiden Erkrankungen. Die Autoren geben eine kurze Übersicht über ambulante, teilstationäre und stationäre Versorgungsangebote und informieren über die Gewährleistung von Rechtsansprüchen.

Insgesamt wird in diesem Buch ein sehr anschaulicher Überblick über die Diagnose und Therapie von Demenz und Depression im Alter gegeben. Den Autoren gelingt es, theoretische Grundlagen, aktuelle Forschungsergebnisse und praxisrelevante Ratschläge in konzeptuell anregender Weise zu verbinden. Damit wird das Buch seinem Anspruch gerecht, die differentialdiagnostische Problematik dieser beiden Krankheiten aufzuzeigen und interessierten Berufsgruppen diagnostische und therapeutische Entscheidungshilfen zu liefern.

Stefanie Brassen, Mannheim

\section{Sigusch (Hrsg.)}

\section{Sexuelle Störungen und ihre Behandlung}

Stuttgart, Thieme, 2001, 3. Auflage, 638 S., $€ 75,67$

ISBN 3-13-103943-4

Die 3. Auflage dieses schon immer vorwiegend psychoanalytisch orientierten Buches wurde erheblich erweitert: Aus dem flexiblen Taschenbuch mit 420 Seiten ist ein großformatiges Hardcover-Werk mit ca. 640 Seiten geworden; damit ist es jetzt fraglos ein Standardwerk der Sexuologie. Alle Kapitel wurden überarbeitet, einige ganz wesentlich erweitert, wie z.B. die Paartherapie bei sexuellen Funktionsstörungen, die männliche homosexuelle Entwicklung und die institutionelle Therapie bei sexueller Delinquenz; 9 von insgesamt 28 Kapiteln sind neu aufgenommen worden. Das Werk ist wieder ein Vielautorenbuch, jedoch stammen 10 Kapitel vom Herausgeber selbst. Dessen Stil hebt sich wohltuend von der üblichen Art trockener Darstellung wissenschaftlicher Inhalte ab; Sigusch schreibt spritzig und frisch, manchmal mit spitzer Feder, und ist dadurch anregend zu lesen. Gleichzeitig sind die von ihm bearbeiteten Inhalte bewundernswert gründlich recherchiert.

Die 3. Auflage umfasst wieder den Bereich der ungestörten und gestörten Sexualität sehr breit und stellt dabei die notwendigen soziokulturellen Bezüge her. Im einleitenden Kapitel «Was heißt sexuelle Störung?» erörtert Sigusch, wie abhängig die Diagnose «sexuelle Störung» von den jeweils geltenden soziokulturellen Normen ist. Anhand eines Rückblicks auf die 2. Auflage aus dem Jahre 1980 macht Sigusch deutlich, wie stark sich manche Ansichten und Haltungen gegenüber der Sexualität allein in den letzten 20 Jahren geändert haben. Im 2. Kapitel setzt er sich mit diesem kulturellen Wandel der Sexualität näher auseinander. Die nächsten 4 Kapitel beschäftigen sich mit der weiblichen und männlichen heterosexuellen und homosexuellen Entwicklung und deren Problemen. Die Autoren setzen sich dabei kritisch mit den frühen psychoanalytischen Ansichten zur sexuellen Entwicklung auseinander, stellen sie neuen Erkenntnissen der Entwicklungspsychologie gegenüber und gehen auf die sich daraus ergebenden Konsequenzen für die Psychotherapie ein.

Der Hauptteil des Buches widmet sich den «sexuellen Symptomen und Störungen» (Kapitel 7-15).

Einleitendend schildert G. Schwind in mehreren Fallvignetten typische Sprechstundensituationen eines Erstgespräches, in denen es meistens darum geht, den Patienten zu helfen, die Funktionalität ihrer sexuellen Symptomatik für ihre Lebenssituation zu erkennen. Im nächsten Kapitel werden die Grundzüge der psychoanalytisch orientierten Einzel- und Paarbe- 
ratung bei Sexual- und Partnerschaftskonflikten (mit Fallbeispielen) besprochen. In den folgenden zwei Kapiteln geht Sigusch sehr detailliert auf die Diagnose und Differentialdiagnose, Symptomatologie, Klassifikation und Epidemiologie sexueller Störungen ein. Er bespricht die Notwendigkeit gezielten Erfragens der Symptomatologie auch bei psychoanalytisch orientierter Arbeitsausrichtung und problematisiert die Trennung von Organo- und Psychogenese sexueller Störungen. In «10 silbernen Erfahrungssätzen» fasst er die sich aus der Anamnese ergebenden differentialdiagnostischen Hinweise für eine vorwiegende Organo- und/oder Psychogenese praxisrelevant zusammen. Danach beschreibt er die sexuellen Störungen anhand der international üblichen Klassifikationsschemata ICD und DSM und kommentiert letztere kritisch. Im anschließenden Kapitel über die Organogenese sexueller Funktionsstörungen geht Sigusch unter anderem auf die schwierige Abklärung ein, ob eine sexuelle Funktionsstörung krankheits-, arzneimittel- und/oder psychisch bedingt ist, eine Abgrenzung, die oft nicht möglich ist.

Das Kapitel 12 (Psychoanalyse und sexuelle Funktionsstörungen) ist eine wissenschaftstheoretische kritische Auseinandersetzung mit der analytischen Sicht insbesondere der weiblichen sexuellen Entwicklung. In Kapitel 13 erläutert Gunter Schmidt recht kurz und knapp die Paartherapie bei sexuellen Störungen und setzt sich mit möglichen Gründen für die Zunahme der sogenannten sexuellen Lustlosigkeit in den letzten Jahren auseinander. Anschließend bespricht Sigusch in seiner charakteristischen kritischen und gründlichen Art die Organotherapie bei sexuellen Funktionsstörungen. Ganz besonders ausführlich stellt er den aktuellen Stand unseres Wissens über die Sildenafil-(Viagra-)Behandlung dar. In den nächsten 3 Kapiteln werden die sexuellen Probleme und Störungen in der gynäkologischen Praxis und bei chronischen Krankheiten sowie psychologische Aspekte der AIDSBehandlung besprochen.

In den folgenden 7 Kapiteln geht es um die Störungen der sexuellen Präferenz bzw. um die sogenannten Paraphilien. Die ersten 2 Abschnitte beschäftigen sich ausschließlich mit der psychoanalytischen Theorie und Therapie von sexuellen Perversionen. Es folgt ein Kapitel über das sehr spezielle Problem der verantwortlichen Abgrenzung pädosexueller Bedürfnisse von sexuellem Kindesmissbrauch und ein Kapitel über die Psychotherapie nach sexueller Traumatisierung. Die Autorin weist auf die Komplexität sexueller Traumatisierungen und die Notwendigkeit hin, die Psychotherapie auf die individuelle Situation des Opfers abzustellen. Zu diesem Thema hätte man sich eine breitere Darstellung gewünscht. Im letzten Kapitel dieses Abschnitts wird unter anderem die grundsätzliche therapeutische Haltung zur Behandlung sexuell Delinquenter diskutiert, ein Überblick über die therapeutischen Möglichkeiten bei in Institutionen untergebrachten Delinquenten gegeben sowie umfassend kritisch über Möglichkeiten und Grenzen somatischer Behandlungen bei sexueller Devianz berichtet.
Zwei Kapitel beschäftigen sich mit den Geschlechtsidentitätsstörungen. Bei der Beschreibung dieser Problematik im Kindes- und Jugendalter wird unter anderem die Schwierigkeit der Abgrenzung einer angenommenen Störung von einer nicht als Störung zu bezeichnenden Geschlechtsrollenhaltung diskutiert, die nur deswegen auffällt, weil sie nicht gesellschaftskonform ist. Anschließend wird ausführlich die Transsexualität als die häufigste und ausgeprägteste Form der Geschlechtsidentitätsstörungen im Erwachsenenalter besprochen, einschließlich der therapeutischen Möglichkeiten und der juristischen Situation.

Den Abschluss bildet ein Bericht über die Entwicklung und den aktuellen Stand der Fort- und Weiterbildung für Sexualmedizin und Sexualtherapie in Deutschland. Die berufsbegleitenden Curricula der Deutschen Gesellschaft für Sexualforschung und der Akademie für Sexualmedizin werden diskutiert.

Das Buch ist nicht «aus einem Guss», mit systematischem Aufbau und einheitlicher Gliederung. Es enthält neben vielen grundsätzlichen sexualmedizinischen und sexualtherapeutischen Darstellungen einige interessante Randkapitel. Viele Abschnitte sind Einzelaufsätze, die für sich gelesen werden können; einige Kapitel behandeln nur grundsätzliche Überlegungen zum Thema, andere geben einen orientierenden Überblick über das derzeitige Wissen und Handeln im jeweiligen Bereich. Die von Sigusch verfassten Kapitel sind besonders umfassend und gründlich bearbeitet. Die üblichen Schwächen eines Vielautorenbuches wie Überschneidungen und Doppeldarstelllungen halten sich in Grenzen. Der unterschiedliche Schreib- und Bearbeitungsstil der Autoren stört nicht; er macht das Werk bunt.

Dieses Buch sollten alle besitzen, die Patienten mit sexuellen Problemen beraten bzw. behandeln. Der Verhaltenstherapeut kann das Grundsätzliche zur Behandlung sexuell gestörter Patienten erfahren und in den psychoanalytisch orientierten Kapiteln andere als verhaltenstherapeutische Sichtweisen kennen lernen.

G. Kockott, München

\section{W. Ecker}

\section{Die Krankheit des Zweifelns}

Lippstadt, Verlag für Psychologie und Lebenshilfe, 1999, 135 S., $€ 14,-$

ISBN 3-980 6209-5-6

Wir alle haben im Laufe unseres Lebens eine Vielzahl von Gewohnheiten und Ritualen entwickelt, die das alltägliche Leben erleichtern, Freiräume für verschiedenste andere Tätigkeiten schaffen und uns Sicherheit geben, nicht jeden Handlungsschritt täglich neu entscheiden zu müssen. Problematisch werden solche Gewohnheiten und Rituale, die sich in Handlungen oder in Gedanken äußern, dann, wenn sie zu einer deutlichen Beeinträchtigung im täglichen Leben führen, 
wenn die Gewohnheiten «entgleisen» und die Berufstätigkeit oder soziale Aktivitäten beeinträchtigen. Man spricht dann von einer Zwangsstörung, deren Kernmerkmal im Grunde der quälende Zweifel ist, ob ein Kontroll- oder Waschritual auch «ordnungsgemäß», «richtig» und «zufriedenstellend» ausgeführt wurde. Der französische Psychiater Pierre Janet hat diese Störung bereits um die Wende vom 19. ins 20. Jahrhundert beschrieben und treffend «la maladie du doute», d.h. die Krankheit des Zweifelns, genannt.

Man kann davon ausgehen, dass etwa 1-2\% der erwachsenen Bevölkerung von einer ernsthaften Zwangsstörung betroffen sind, d.h. etwa 1 Million der Bevölkerung in Deutschland leiden unter einer ernsthaften Zwangsstörung, die «Krankheit des Zweifelns» ist also in der psychotherapeutischen Versorgung ein ernstes Problem.

Aus der Praxis ist bekannt, dass Zwangsstörungen langfristig und dauerhaft nur dann deutlich zu bessern sind, wenn bei den Betroffenen eine hohe Motivation zur aktiven Mitarbeit und zur aktiven Überwindung der eigenen «schlechten» Gewohnheiten und Rituale vorliegt und wenn die Bereitschaft zur Teilnahme an einer Selbsthilfegruppe gegeben ist.

So hat Willi Ecker ein kurzgefasstes, gut lesbares, verständlich geschriebenes Buch primär für Betroffene und deren Angehörige vorgelegt.

Nach der Einleitung ist es in zwei umfangreichere Teile, nämlich Teil I: «Grundlegende Informationen zur Zwangsstörung» und Teil II: «Möglichkeiten der Behandlung» gegliedert.

Im 1. Teil wird geklärt, was eine Zwangsstörung ist, wie man sie gegen andere Erkrankungen wie Depression, schizophrene Psychose, hirnorganische Erkrankung, überwertige Ideen, pathologisches Spielen oder zwanghafte Persönlichkeit abgrenzen kann und welche Ursachen der Zwangsstörung bekannt sind. Es ist nicht leicht für den Rezensenten, sich in die Verständnisbereitschaft eines Laien hineinzuversetzen. In diesem Teil werden meines Erachtens zu stark Kenntnisse über Fachbegriffe wie beispielsweise Wahnphänomene oder Vorwissen über die erwähnten Begleiterkrankungen vorausgesetzt. Dieser recht kurze Teil hätte durchaus ein wenig ausführlicher und für Laien verständlicher abgefasst werden können.

Im 2. Teil werden ausführlich und verständlich Möglichkeiten der Behandlung beschrieben. Man spürt dabei deutlich die reichhaltige persönliche Behandlungserfahrung des Autors. Alle Behandlungsansätze werden anschaulich durch Fallbeispiele illustriert. Es wird immer wieder darauf hingewiesen, dass der Betroffene im Zweifelsfall Rücksprache mit einem Therapeuten nehmen sollte. Vielleicht hätte man in diesem Teil noch deutlicher darauf hinweisen können, dass eine Selbstbehandlung nur dann letztlich erfolgreich sein kann, wenn man sich mit hoher Selbstdisziplin auch an die Vorgaben dieses Buches hält und wenn der/die Betroffene sein/ihr individuelles Behandlungskonzept entwickelt und eine Selbstbehandlung über einen längeren Zeitraum - im Buch wird einmal, jedoch nur nebenbei, ein Zeitraum von 6 Monaten erwähnt - durchhält.
Alle Behandlungsformen und unterschiedlichen Vorgehensweisen zur Therapie der Zwangsgedanken und zur Therapie von Kontroll- und Waschzwängen wurden angesprochen. Sehr gut ist im Zusammenhang mit der Behandlung von Kontrollzwängen die Erwähnung und Beschreibung des «Unvollständigkeitsgefühls».

Es handelt sich um ein Buch, das dem Anliegen, «eine psychologische Lebenshilfe» zu geben, gerecht wird. Deshalb ist ihm eine breite Leserschaft unter den Betroffenen zu wünschen. Vielleicht sollte man aber als Therapeut/Therapeutin doch einige Punkte beachten, wenn man dieses Buch als Begleittext einer Zwangsbehandlung empfiehlt. Es hätte ein wenig deutlicher herausgestrichen werden können, wo die Grenze zwischen einer noch nicht behandlungsbedürftigen, tolerierbaren «Marotte» und einer krankheitswertigen Zwangsstörung liegt und dass Selbsthilfe erfolgreicher ist, wenn man ein Behandlungsprogramm, zu dem dieses Buch ein hervorragender Begleittext sein kann, unter professioneller therapeutischer Hilfe beginnt und nach einer begrenzten Zahl von Behandlungskontakten auf eine Selbsthilfe übergeht. Mit diesem Buch kann man meines Erachtens die Behandlung einer schweren Zwangsstörung durch einen erfahrenen Psychotherapeuten nicht ersetzen. In diesem Sinne ist aber dem Buch ein Erfolg zu wünschen.

Eibe-Rudolf Rey, Mannheim

\section{B. Peters \\ Psychotherapie auf dem Prüfstand. Über Sitten und Gebräuche in helfenden Berufen}

Heidelberg, Asanger, 2001, 163 S., € 25,-

ISBN 3-89334-361-X

Ein neues Buch über Psychotherapie - folglich ein weiterer Beitrag zum Dauer-Modethema «Neue Wege in der Psychotherapie»? Die Antwort lautet: Ja und Nein! Ja, denn Bruno Peters nutzt jede einzelne Seite und alle schriftstellerischen Mittel, um uns «Helfern, Therapeuten, Erziehern und anderen Gutmenschen» neue Sicht- und Herangehensweisen an das «Geschäft des Heilens» nahezubringen. Nein, denn im Gegensatz zu den meisten anderen modernen Publikationen zum Thema fokussiert Peters nicht die KlientInnen mit ihren spezifischen Symptomatiken, die neue spezialisierte Hilfsangebote erfordern, sondern richtet sein Augenmerk in erster Linie auf die Wege der TherapeutInnen selbst, die - zu seinem großen Bedauern - «sehr darauf [achten], in den heiligen Hallen ihres Theoriegebäudes umherzuwandeln, ... in denen der heilige Atem der Methode weht und alle Umherwandelnden dieselbe Sprache sprechen» (S. 7).

Nachdem Peters derart gleich zu Beginn die orthodoxen VertreterInnen aller Therapieschulen vom Thron gestoßen hat, proklamiert er im Folgenden eine Art «therapeutischen Narrentums», dessen Grundsäulen er auf äußerst anschauliche Art und Weise mit zahlreichen Fallbeispielen, Geschichten 
sowie anregenden Bezügen zur Theaterwissenschaft (Peters ist «im zweiten Leben» Schauspieler!) illustriert. Neu sind dabei weniger diese Grundsäulen selbst, denn mit Begriffen wie «Ressourcenorientierung», «Humor» oder «Respektlosigkeit gegenüber traditionellen therapeutischen Methoden» dürften mittlerweile alle PsychotherapeutInnen vertraut sein. Neu sind vielmehr die Selbstverständlichkeit und die Radikalität, mit der Peters die Nutzung der individuellen Ressourcen der TherapeutInnen (Kreativität, Humor, eigene Gefühle, Bilder, erste Eindrücke usw.) als Mittel der Befreiung von den so weit verbreiteten Mechanismen der «therapeutischen Selbstbehinderung» und der «erlernten Inkompetenz» propagiert. Trotz des Fehlens einer erkennbaren Systematik schafft es Peters, Schlagwörtern wie «Salutogenese», «therapeutisches Theater» oder «Humor und kreative Lösungen in der Therapie» frisches Leben einzuhauchen. Besonders interessant sind dabei seine Überlegungen zum therapeutischen Hang zur Problemfixierung und zu der Frage, wie dieser zugunsten einer Ressourcen- und Lösungsorientierung überwunden werden kann - wobei Peters aber keineswegs an schnellen, oberflächlichen Lösungen interessiert ist! Erfreulich ist auch seine Entscheidung, im Anhang einen Vortrag von Dr. Wolfgang Neumann abzudrucken, der mit Hilfe einiger anschaulicher Fallbeispiele die praktische Relevanz der therapeutischen Grundvariable «Ressourcenorientierung» noch einmal eindrucksvoll darlegt.

An Bruno Peters' Buch werden sich vermutlich die Geister scheiden, denn es ist eines jener wenigen Bücher, die tatsächlich das einlösen, was sie im Vorwort versprechen - und zwar im positiven wie im negativen Sinne: So schreibt Peters etwa in seiner Einleitung, dass er «wie in einer Art Kaleidoskop zeigen [will, wie] ... das Geschäft des Heilens ... auch betrachtet ... werden kann» und dabei «keinen Anspruch auf Vollständigkeit und Systematik» (S. 3f.) erhebt. Treffender könnte er sein Buch gar nicht charakterisieren! Folglich hätte der Verlag meines Erachtens auch gut daran getan, den vom Autor im Vorwort erwähnten Titel «Ansichten eines therapeutischen Narren» zu übernehmen, da dieser deutlich besser zum Ausdruck gebracht hätte, dass es sich bei dem Buch um die (fach-)literarisch angereicherten Ideen und Überlegungen eines erfahrenen Therapeuten und Supervisors handelt, nicht aber um eine systematische Analyse bestehender psychotherapeutischer Verfahren.

Auch Peters' vorab geäußerte Zweifel, ob es ihm gelungen sei, «die Regeln des akademischen Arbeitens einzuhalten» (S. 4), stellen sich leider nur allzu schnell als sehr realistisch heraus: Kaum ein Zitat ist zweifelsfrei als solches zu erkennen, auf Seitenzahlangaben verzichtet der Autor gänzlich, und wenngleich Quellen meist genannt werden, bleibt doch oft unklar, wer nun genau was gesagt hat! Die Chancen schließlich, eine im Text angeführte Arbeit auch tatsächlich im Literaturverzeichnis zu finden, liegen etwa bei fifty-fifty! Sollten Sie also in Erwägung ziehen, dieses Buch als Grundlage für eine Literaturrecherche - etwa zum Thema «Ressourcenorientierung» oder «Humor»- zu Rate zu ziehen, so dürfte es Ihnen etwa so nützlich sein wie eine Diplomarbeit über die Beziehung von Donald Duck zu seinem Onkel Dagobert.

Das wissenschaftliche Arbeiten scheint Peters' Sache also nicht zu sein - das wird auch in dem (glücklicherweise sehr kurzen) Kapitel über «Kreativität und Ressourcensensibilität» offenkundig, das sich mit seinem bemüht-theoretisierenden Duktus deutlich negativ vom Rest des Buches abhebt. Nein, stark ist Peters immer dann, wenn er - wie meist - den weisen Narren gibt und uns an seinen aus langjähriger therapeutischer Praxis gewonnenen Erfahrungen teilhaben lässt, an seinen «närrischen Interventionen», seinem Balanceakt zwischen menschlichem Respekt und therapeutischer Respektlosigkeit oder seinem Wissen über das Theater und dessen Nutzbarmachung für die Psychotherapie.

Bruno Peters hat ein Buch über Psychotherapie geschrieben und wie in einer Therapie kam ich mir auch oft beim Lesen vor: Es gab Höhen und Tiefen, Plattitüden und Geistesblitze, Momente, in denen ich das Gefühl hatte, mir würde aus dem Herzen gesprochen, und Passagen, in denen ich nicht verstand oder schlicht befremdet war. Es gab Phasen, in denen scheinbar gar nichts und dann plötzlich wieder ganz viel passierte. Es gab keinen offensichtlichen roten Faden und doch immer wieder den Rückbezug auf die entscheidenden Themen. Und es gab Emotionen: ich war berührt und erfreut, verärgert und frustriert, manchmal habe ich laut gelacht und genauso oft laut geflucht! Gegen Ende dann begannen die Dinge sich zu wiederholen (das ist durchaus wörtlich gemeint, denn die letzten 6 Absätze des Textes entsprechen fast identisch einem Abschnitt auf Seite 54f.!), so dass ich mit dem Gefühl abschließen konnte, hier jetzt alles gelernt zu haben, was es zu diesem Zeitpunkt zu lernen gab!

Ob mir das Buch nun auch helfen wird bei meinen eigenen therapeutischen Hilfsversuchen? Ich weiß es nicht! Aber auf jeden Fall hat es mich bewegt - und das ist ja dann nicht weit entfernt von Peters' Erfolgs-Definition: «Ich habe Erfolg, wenn sich im System des Klienten etwas 〈bewegt〉, eine «Lösung> sichtbar wird.» (S. 109)

Björn Süfke, Bielefeld

\section{A. Kobelt, E.V. Grosch, F. Lamprecht \\ Ambulante psychosomatische Nachsorge - Integratives \\ Trainingsprogramm nach stationärer Rehabilitation}

Gewürdigt mit dem Rehabilitationsförderpreis

Stuttgart, Schattauer, 2001, 258 S., $€ 30,95$

ISBN 3-7945-2138-2

Brauchen wir ein eigenes Therapiemanual für die ambulante psychosomatische Nachsorge? Wird da nicht alter Wein in neue Schläuche gegossen? Solche und ähnliche Fragen gehen einem durch den Kopf, wenn man das Trainingsprogramm von Kobelt et al. das erste Mal in Händen hält. Doch schon bald nachdem einige Kapitel gelesen sind, wird klar, dass dieses 
Buch weit mehr ist als ein weiteres Therapeutenmanual für den Bücherschrank.

Stationäre psychosomatische Rehabilitation bedeutet für viele Patienten vorübergehende Entlastung von alltäglichen Verpflichtungen mit der Möglichkeit, im geschützten Rahmen neue Verhaltensweisen auszuprobieren und durch emotionale Öffnung neue Erfahrungen zu sammeln. Außerhalb der geschützten Umgebung zeigt sich jedoch oft, dass viele der gemachten Erfahrungen den Alltagsbelastungen noch nicht standhalten. Die Verbesserung des Übergangs von stationärer Therapie in den Alltag ist ein Thema, dessen sich Kostenträger und Versorger in letzter Zeit in Form von Nachsorge- und Vernetzungskonzepten verstärkt angenommen haben.

1994 erhielt die Arbeitsgruppe um Herrn Professor Lamprecht, Abteilung für Psychosomatik und Psychotherapie der Medizinischen Hochschule Hannover (MHH), von der Landesversicherungsanstalt (LVA) Hannover den Auftrag, Nachsorgegruppen, die sich aus Rehabilitanden der LVA zusammensetzten, zu versorgen. Dabei sollte ein Rahmen geschaffen werden, der Raum für neue Erfahrungen und schulen- bzw. professionsübergreifende Interventionsmöglichkeiten lässt.

Im Vordergrund stand die Hilfestellung bei der Implementation dessen, was die Betroffenen für sich in der Rehabilitationsklinik erarbeitet hatten. Es sollte sich ausdrücklich nicht um eine alleinige Fortsetzung der psychotherapeutischen Maßnahmen der Rehabilitationsklinik handeln.

Der siebenjährige Erfahrungsschatz dieser Arbeitsgruppe wird im Trainingsprogramm von Kobelt und Mitautoren auf das Wesentliche konzentriert und macht dieses Buch so zu einem wertvollen Begleiter für alle, die sich dem Thema «Alltagsrelevanz von psychotherapeutischen Interventionen» widmen.

Die Autoren gehen in einem kurzen Einführungsteil zunächst auf die allgemeinen Probleme beim stationär-ambulanten Übergang und auf Aspekte, die die Durchführung des Nachsorgeprogramm betreffen, ein. Bereits hier fallen die vielen praxisrelevanten Themen ins Auge, welche Schnittstellenprobleme ebenso erfassen wie Gruppenleiterverhalten und eine kurze Diskussion der Vor- und Nachteile einer manualisierten Vorgehensweise. Im Anschluß werden 14 Module im Sinne eines Therapeutenleitfadens für gruppentherapeutische Sitzungen vorgestellt. Nach einer Einführungssitzung beginnt die Nachsorge inhaltlich mit dem Thema «Abschied von der Rehabilitationsklinik - Stillstand, Veränderung oder Rückkehr zum Ausgangspunkt». Es folgen störungsspezifische Module zu den Bereichen Angst, Depression und funktionelle Beschwerden. Die weiteren Einheiten umfassen Themen wie Probleme am Arbeitsplatz, Beziehungsprobleme, Identifikation von Veränderungswiderständen, Aufbau von Ressourcen, Verhalten in kritischen Situationen, Misserfolg und eigenverantwortliches Handeln. Am Ende findet sich ein Modul zum Thema Psychopharmaka.

Gewissermaßen als Fahrplan für erfahrene Gruppenleiter befindet sich am Anfang eines jeden Moduls ein Kurzüberblick mit Zielsetzung, Vorschlägen für die Eröffnung der Sitzung, möglichen Gruppenthemen, Interventionen, Abschluss der Sitzung und Hinweisen zu spezifischen gruppendynamischen Aspekten. Die einzelnen Bereiche werden anschließend im Text ausführlich dargestellt und mit Fallbeispielen illustriert. Die störungsspezifischen Einheiten enthalten zu Beginn eine -allerdings sehr knapp gehaltene - theoretische Einführung in psychoanalytische und lerntheoretische Modelle. Mögen diese Einführungen auch zu kurz gehalten sein, so wird man durch die vielen praxisrelevanten Hinweise und Themen doch mehr als entschädigt. Beispielsweise finden sich Interventionen mit dem Titel «Wie kann ich jemandem verzeihen, dem ich einfach nicht vergeben kann?» oder «Wie wichtig ist mir mein Partner wirklich, wenn ich mich nicht entscheiden kann, ob ich mit ihm oder ohne ihn leben möchte?»

Einen wichtigen Bereich stellen dabei auch Themen aus der Arbeitswelt des Patienten dar. Neben den wichtigsten grundlegenden Informationen zu Erwerbsminderung und berufsfördernden Leistungen zur Rehabilitation enthält dieses Modul eine Reihe von Themen aus dem Berufsalltag wie z.B. Umgang mit Konflikten mit Arbeitskollegen, Verhalten bei Mobbing, Umgang mit Abgrenzung und individuelles Anforderungsprofil am Arbeitsplatz.

Bei allen Vorbehalten gegenüber Typologien können die von den Autoren am Ende des Buches dargestellten Typologien eine wichtige Hilfe im Umgang mit typischen Interaktionsmustern liefern. Hier zeigt sich erneut die Stärke dieses Buches, das vorwiegend aus der Praxis geboren wurde. Man findet Hinweise zum Umgang mit Rentenbegehrern, mit somatisch fixierten, äußerlich nicht als belastet erkennbaren oder mit enttäuschten, überraschten, duldsamen, solidarischen, ängstlichen oder trauernden und kulturfremden Patienten. Respekt und Verständnis für die Erlebensweise der Patienten bleiben dabei stets gewahrt.

Am Ende des Buches wird ein kurzer Überblick über die Ergebnisse der von den Autoren Kobelt und Lamprecht begleitend zum Therapieprogramm durchgeführten Evaluationsstudie gegeben.

Im Anhang stellen die Autoren zahlreiche Materialien zur Verfügung, angefangen vom Text für eine Patientenbroschüre über einen Textvorschlag für eine Phantasiereise bis hin zu zahlreichen Schaubildern und Problemstrukturierungshilfen. «Ambulante psychosomatische Nachsorge» ist ein Therapeutenmanual, das durch seine pragmatische, praxisnahe Konzeption besticht und inhaltlich durch seine Ausrichtung auf die speziellen Aspekte der Nachsorge eine längst überfällige Lücke schließt.

$\mathrm{Zu}$ Recht ist der Erstautor Dr. Dipl.-Psych. Axel Kobelt für die Erarbeitung des Manuals und die Evaluation des Behandlungskonzeptes mit dem Rehabilitationsförderpreis 2000 der Karlsruher Sanatorium-AG auf dem jährlich stattfindenden Rehabilitationskolloquium des VDR ausgezeichnet worden.

Bernhard Osen, Prien am Chiemsee 